DIFFICULT TO DIAGNOSE SLE MANIFESTATIONS ASSOCIATED WITH CARDIAC ARREST, MYOCARDITIS, CHRONIC PERICARDITIS, POLYNEUROPATHY

Tatjana Zekić. Dept. of rheumatology and clinical immunology, University Hospital Rijeka, Rijeka, Croatia

10.1136/lupus-2020-eurolupus.97

Introduction Cardiac manifestations and polyneuropathy in lupus are common, although clinically manifest myocarditis is rare, estimated at $9 \%$.

Case report Female patient 44 years old came to the Emergency Department due to general weakness and dizziness. She has lost $14 \mathrm{~kg}$ in the last 3 months, she has subfebrility, fatigue, hair loss, joint pain. A cardiac arrest with ventricular fibrillation (VF) occurred during the examination. Successful resuscitation was performed. Echocardiography indicates an ejection fraction (EF) of $5 \%$ and contractility disorder. Mechanical circulatory support to venous-arterial extracorporeal membrane oxygenation (V-A ECMO) was introduced. Extensive cardiac treatment and work up was done, coronarography (normal), myocardial biopsy (lymphocytic myocarditis less likely gigantocellular), pericardial biopsy (chronic pericarditis), lysis of pericardial adhesion with improvement in EF. Cardiac MRI verifies diffuse inflammatory myopericarditis. The serology on Chlamidophila pneumonie was positive. Tetracycline therapy was started. Asymmetric tetraparesis occurred during cardiac treatment, muscle biopsy was 
without inflammatory infiltrate, cervical and thoracic spine MR was normal, EMNG indicated polyneuropathy, and she was diagnosed with Critical illness polyneuropathy. There was a suspected ischemic lesion on the brain MR temporoparietally to the right. Thoracic CT was normal. In that moment, she was transferred to the Department of Immunology. The treatment was started according to cardiac guidelines for myocarditis, solumedrol $1 \mathrm{mg} / \mathrm{kg}$, and $90 \mathrm{mg}$ IVIG for 3 days after which she started recovering neuromuscular symptoms. Of the SLICC criteria she had nonscarring alopecia, arthritis, serositis, positive ANA, 1:320, homogenous, ds DNA, low complement (C3, C4). In maintenance therapy, she has a low dose of glucocorticoids, azathioprine $100 \mathrm{mg}$ and has been in remission for 2 years.

Conclusion The patient had complications in unrecognized systemic lupus, critical illness polyneuropathy, infectious myocarditis and chronic constrictive pericarditis, who recovered only from cardiac support and medication. Accurate diagnosis in SLE-mimicking symptoms is possible with extensive diagnostics.

\section{P51 TRENDS IN MORTALITY IN SYSTEMIC LUPUS ERYTHEMATOSUS: AN ANALYSIS OF SLE INPATIENT MORTALITY AT UNIVERSITY HOSPITAL COVENTRY AND WARWICKSHIRE NHS TRUST FROM 2007-2016}

${ }^{1}$ Himashi Anver, ${ }^{2}$ Shirish Dubey, ${ }^{3}$ James Fox. ${ }^{1}$ Dept. of Rheumatology, Poole General Hospital, Poole; ${ }^{2}$ Dept. of Rheumatology, University Hospital Coventry and Warwickshire, Coventry; ${ }^{3}$ Emergency Dept., Wirral Teaching Hospital, Birkenhead, UK

\subsection{6/lupus-2020-eurolupus.98}

Background The aim of this study was to determine the causes of mortality in patients with systemic lupus erythematosus (SLE) at the University Hospital Coventry and Warwickshire (UHCW) NHS Trust over a $10 y r$ period.

Methods This was a retrospective study of patients who had died in UHCW NHS Trust between 2007 and 2016, where SLE or lupus was mentioned on the death certificate. Ethics approval was obtained from the Research and Development.

Results We identified 22 patients out of 1979 patients with SLE who had died during the period between 2007- 2016, 7 of these patients were under 50. The leading cause of death was infection. Active disease was associated with higher mortality and younger age of death. We identified 3 patients with biopsy proven lupus nephritis and 1 patient with CNS lupus. Median age at death was 58.5 years, with median duration of disease of 14.5 years. Constitutional symptoms were the main symptoms of system involvement found in our study population, seen in 68.2\%. Surprisingly, none of the patients died because of vascular problems.

Conclusion The study suggests a changing trend in SLE mortality with none of the deaths in this cohort being due to cardiovascular or cerebrovascular disease. Infection continues to be the biggest reason for mortality in this cohort.

Note This abstract is due to be published as a full paper in the Rheumatology International Journal.

\section{P52 THE RELATIONSHIP OF VITAMIN D LEVELS WITH DISEASE ACTIVITY IN SYSTEMIC LUPUS ERYTHEMATOSUS}

'Lambros Athanassiou, ${ }^{2}$ Ifigenia Kostoglou, ${ }^{3}$ Pavlos Tsakiridis, ${ }^{3}$ Aikaterini Tzanavari, ${ }^{3}$ Eirini Devetzi, ${ }^{3}$ Marina Gatsiou, ${ }^{4}$ Michael Koutsilieris, ${ }^{3}$ Panagiotis Athanassiou. ${ }^{1}$ First Dept. of Medicine, Asclepeion Hospital, Voula, Athens; ${ }^{2}$ Dept. of Endocrinology, Asclepeion Hospital, Voula, Athens; ${ }^{3}$ Dept. of Rheumatology, St. Paul's Hospital, Thessaloniki; ${ }^{4}$ Dept. of Physiology, Medical School, University of Athens, Athens, Greece

\subsection{6/lupus-2020-eurolupus.99}

Background Vitamin D is known to exert a potent immunomodulatory action. It has immunostimulatory properties and in parallel exerts immunosuppression and inhibits tissue destruction related to autoimmunity. Vitamin D deficiency, thus, is related to immune activation and the development of autoimmune diseases. Systemic lupus erythematosus (SLE) is an autoimmune disease affecting all organ systems. It develops in all age groups, however young female patients are particularly vulnerable. Vitamin D status is currently assessed by the measurement of $25(\mathrm{OH}) \mathrm{D}_{3}$ levels. The aim was to assess vitamin D levels in a cohort of SLE patients and examine the relationship of vitamin $\mathrm{D}$ levels with disease activity. Measurements were also performed in a control group.

Methods In a cohort of 30 SLE patients, 27 female and 3 male and a control group $25(\mathrm{OH}) \mathrm{D}_{3}$ levels were measured. In all SLE patients clinical and laboratory examination was performed to assess the activity of the disease. Serum inflammation markers were measured. $25(\mathrm{OH}) \mathrm{D}_{3}$ levels were measured by radioimmunoassay. The measurement of $25(\mathrm{OH}) \mathrm{D}_{3}$ was performed in a two-step procedure. The first step aimed at rapid extraction of $25(\mathrm{OH}) \mathrm{D}$ and other hydroxylated metabolites from serum or plasma with acetonitrile. Following extraction, samples were assayed by competitive RIA using an antibody with specificity to 25OHD. The sample, antibody and tracer were incubated for $90 \mathrm{~min}$ at $20-25^{\circ} \mathrm{C}$. Phase separation was accomplished after $20 \mathrm{~min}$ incubation at 20-25 ${ }^{\circ} \mathrm{C}$ with a second antibody precipitating complex. The sensitivity of the assay was $<1.6 \mathrm{ng} / \mathrm{ml}$ and the recovery approximately $100 \%$ for $25(\mathrm{OH}) \mathrm{D}_{3}$. Within and between batch precision was $<12 \%$ and $<11 \%$, respectively.

Results Serum $25(\mathrm{OH}) \mathrm{D}_{3}$ levels were significantly lower in SLE patients than in the control group $(\mathrm{p}<0.001$, Student's $\mathrm{t}$ test). An inverse relationship was observed between inflammation markers and $25(\mathrm{OH}) \mathrm{D}_{3}$ levels, lower $25(\mathrm{OH})$ $\mathrm{D}_{3}$ levels observed in SLE patients with high inflammation markers.

Conclusions Vitamin D insufficiency or frank deficiency has been observed in patients with autoimmune diseases, such as rheumatoid arthritis. Low vitamin D levels have also been observed in SLE patients. In the described cohort of SLE patients low vitamin D levels were observed and there was an inverse relationship between vitamin D levels and disease activity. In view of the fact that SLE patients display photosensitivity and are advised to avoid sun exposure, these findings denote that $25(\mathrm{OH}) \mathrm{D}_{3}$ levels should be measured in SLE and that patients should be supplemented with cholecalciferol as the hormone has immune modulating properties. 